INSTITUT NATIONAL DE RECHERCHE EN INFORMATIQUE ET EN AUTOMATIQUE

\title{
A class of piecewise linear differential equations arising in biological models
}

\author{
Jean-Luc Gouzé — Tewfik Sari
}

$\mathbf{N}^{\circ} 4207$

Juin 2001

THÈME 4 



\title{
A class of piecewise linear differential equations arising in biological models *
}

\author{
Jean-Luc Gouzé , Tewfik Sari ${ }^{\dagger}$ \\ Thème 4 - Simulation et optimisation \\ de systèmes complexes \\ Projet Comore \\ Rapport de recherche $n^{\circ} 4207$ - Juin 2001 - 19 pages
}

\begin{abstract}
We investigate the properties of the solutions of a class of piecewise-linear differential equations. The equations are appropriate to model biological systems in which there are switch-like interactions between the elements of the network. The analysis uses the concept of Filippov solutions of discontinuous righthand sides equations. It gives an insight on the so-called singular solutions which lie on the surfaces of discontinuity. We study several examples of genetic networks.
\end{abstract}

Key-words: Discontinuous differential equations, steady states, Filippov solutions, stability, biological systems, genetic networks.

\footnotetext{
* Supported by the GdR CNRS Automatique and the French COREV group

$\dagger$ Permanent adress : Laboratoire de Mathématiques, 4 rue des Frères Lumière, 68093 Mulhouse, France. E-Mail: T.Sari@univ-mulhouse.fr
} 


\section{Une classe d'équations différentielles linéaires par morceaux provenant de modèles biologiques}

Résumé : Nous étudions une classe de systèmes différentiels linéaires par morceaux, qui interviennent notamment quand les interactions entre éléments d'un réseau biologique sont du type "à seuil". Ces modèles sont très fréquemment utilisés dans les domaines des réseaux enzymatiques ou génétiques, et ont fait l'objet de nombreux travaux. Notre analyse utilise le concept de solutions de Filippov pour des équations à second membre discontinu. Nous montrons que l'étude des solutions singulières, sur les surfaces de discontinuité, est clarifiée, et nous étudions plusieurs exemples de réseaux génétiques.

Mots-clés : Equations différentielles discontinues, équilibres, solutions de Filippov, stabilité, systèmes biologiques, réseaux génétiques. 


\section{Contents}

1 Introduction $\quad 4$

$\begin{array}{lll}2 & \text { The state transition graph } & 6\end{array}$

3 Filippov solutions of the PL system $\quad 7$

4 Regular and singular stationary points $\quad 9$

5 Examples $\quad \mathbf{1 0}$

5.1 Example of Plahte, Mestl and Omholt $[7] \ldots \ldots \ldots$

5.2 Example of Snoussi and Thomas $[10] \ldots \ldots \ldots \ldots \ldots$

5.3 Example of Thomas $[7] \ldots \ldots \ldots \ldots \ldots \ldots$

6 Conclusion 13

\section{List of Figures}

1 The velocity of motion along a threshold hyperplane. . . . . . . . . . . . 15

2 The velocity of motion along the intersection of two threshold hyperplanes. . 16

3 The flow and transition graph of $(11) \ldots \ldots \ldots \ldots \ldots \ldots$

4 The flow and transition graph of $(12) \ldots \ldots \ldots \ldots \ldots$

5 The transition graph of $(13) \ldots \ldots \ldots \ldots \ldots$

6 The domains of existence of SSP of (13 and 16), shown in the $g_{2}-g_{3}$ plane for an arbitrary value of $g_{1} \ldots \ldots \ldots \ldots \ldots \ldots$ 


\section{Introduction}

Complex biological systems are often modelled with switch-like interactions between the variables, involving step functions. For example, such a behaviour involving thresholds has been shown from an experimental point of view in enzymatic or genetic networks: in the model, the rate of production of the enzyme (or gene) is often described by a sigmoid function, such that the Hill function $\Sigma(\xi, \theta, p)=\xi^{p} /\left(\xi^{p}+\theta^{p}\right)$, where the threshold is $\theta$. If $p$ is large, the function is similar to a step function. The general equations of the model can be written as (cf. [7]):

$$
x_{i}^{\prime}=f_{i}(x)-\gamma_{i} x_{i} \quad i=1, \cdots, n
$$

where the relative degradation rate $\gamma_{i}$ of the component $x_{i}$ is constant positive, and the production rate function $f_{i}$ depends on the $n$ components $x=\left(x_{1}, \cdots, x_{n}\right)$. We assume that $f_{i}$ are piecewise constant positive functions whose values change as the variables $x_{i}$ crosses certain thresholds (cf. the more precise description below). This system has been widely studied in the literature, mainly in the framework of genetic networks (cf. $[4,11])$. In fact, it is a piecewise linear differential system of a particular kind, and the dynamical behaviour of the variables in each of the boxes between thresholds is simple: therefore, the description of the behaviour can be linked to Boolean automata, involving Boolean variables (corresponding to the continuous variable below or above the threshold). Many other problems arise and a lot of interesting work has been done in this domain (for more details see $[7,11])$.

From a mathematical point of view, these systems are differential systems with discontinuous dynamics, and one of our goals in this paper is to show that the concept of Filippov solutions ([3]) can clarify and make easier the study of such systems, in particular of the behaviour on the threshold planes.

Let us introduce some notations: we assume that, for each variable $x_{i}, i=1, \cdots, n$ there are $N_{i}$ thresholds

$$
0<\theta_{i}^{1}<\cdots<\theta_{i}^{N_{i}}<+\infty
$$

which divide the positive cone $C=\left\{x: x_{i} \geq 0, i=1, \cdots, n\right\}$ into $N=\left(N_{1}+1\right) \cdots\left(N_{n}+1\right)$ boxes, of dimension $n$, separated by the $(n-1)$-dimensional hyperplanes $x_{i}=\theta_{i}^{j}$. We assume that the $f_{i}$ are constant positive on each box. The values of $f_{i}$ differ from box to box, so that system (1) is a piecewise linear (PL) system. In vector notation (1) rewrites as

$$
x^{\prime}=f(x)-\gamma x, \quad x \in C, \quad f: C \rightarrow \mathbb{R}^{n},
$$

where $f=\left(f_{1}, \cdots, f_{n}\right)$ and $\gamma=\operatorname{diag}\left(\gamma_{1}, \cdots, \gamma_{n}\right)$, where diag is the diagonal matrix built on the vector.

In practice (2) often involve step functions and is given as

$$
x^{\prime}=F(z(x))-\gamma x,
$$


where $F: \mathbb{R}^{N_{1}+\cdots+N_{n}} \rightarrow \mathbb{R}^{n}$ is of class $\mathcal{C}^{1}$, and

$$
z(x)=\left(z_{1}^{1}, \cdots, z_{1}^{N_{1}}, \cdots, z_{n}^{1}, \cdots, z_{n}^{N_{n}}\right), \quad z_{i}^{j}=S\left(x_{i}, \theta_{i}^{j}\right) .
$$

Here $S(\xi, \theta)$ is the step function

$$
S(\xi, \theta)=\left\{\begin{array}{lll}
0 & \text { if } & \xi<\theta \\
1 & \text { if } \quad \xi>\theta .
\end{array}\right.
$$

In the interior of each box, the behaviour is linear and simple; the problems arise on the separating hyperplanes. In particular, a definition is needed for the singular steady points located on the thresholds (because the vector field is not defined on these surfaces).

Two different methods were developed for the study of the stationary points which are located on threshold hyperplanes. The first method [10] uses logical variables, while the second method $[7,8]$ uses a continuous homologous system of the PL system (see Section 4 for some details). For example, the Hill function $\Sigma(\xi, \theta, p)=\xi^{p} /\left(\xi^{p}+\theta^{p}\right)$ approaches the step function when $p \rightarrow+\infty$. It was often used to define continuous homologues of the PL system $[1,2,4]$. Other functions may be used, like the logoid function [7]. The system becomes now an ordinary differential equations with continuous right hand side, and the steady points are defined in the usual way. But when one comes to the limit of the step functions, it is not so simple to obtain rigorous results on the steady points and their nature.

Moreover, one remaining problem is that of the behaviour of the system on the threshold hyperplanes (and far from a steady state). In fact, the literature on system (2) is concerned mainly with the non-singular solutions (we call non-singular solutions $x(t)$ of $(2)$ solutions in which for each $i, x_{i}(t)=\theta_{i}^{j}$ only at isolated values of $t$ ). The problem of the behaviour on the threshold hyperplanes (called walls in [7]) is not clearly elucidated; this problem appears if the trajectories approach the wall from both sides (black wall). Even if the trajectory is well defined and crosses the wall at a point where one variable only is at the threshold (e.g. transparent walls in [7]), a more complex behaviour can occur in the intersection of two or more threshold hyperplanes, and the solution can be singular in this case also.

The aim of this paper is to study system (2) and to describe its singular solutions. Our approach will be to use the Filippov solutions of a discontinuous differential equations; the definition of these solutions enables to define afterwards, in a clear and easy way, the singular steady points and solutions.

The paper is organised as follows. In Section 2, we describe the transition graph associated to the PL system (2). In Section 3 we define the PL system on the threshold planes and we consider its solutions in the sense of Filippov. In Section 4 we discuss the regular and singular stationary points and we clarify some results in the literature. Section 5 illustrates our approach on some examples which were considered by several authors; we show the applicability of the approach and its simplicity to solve unresolved problems. 


\section{The state transition graph}

Let $q_{i} \in\left[0, N_{i}\right], i=1, \cdots, n$ be integers. Consider the (open) $n$-box

$$
B=B^{q_{1} . . q_{n}}:=\left\{x \in C: \theta_{i}^{q_{i}}<x_{i}<\theta_{i}^{q_{i}+1}, i=1, \cdots, n\right\},
$$

with the special cases $0 \leq x_{i}<\theta_{i}^{1}$ when $q_{i}=0$ and $\theta_{i}^{N_{i}}<x_{i}<+\infty$ when $q_{i}=N_{i}$. The value of $f$ in this box is denoted by $f^{B}$ or $f^{q_{1} . . q_{n}}$.

Theorem 1 In the box (4), the solution $x(t)$ of (2) satisfying $x\left(t_{0}\right) \in B$, is

$$
x(t)=\Phi^{B}+\left(x\left(t_{0}\right)-\Phi^{B}\right) \mathrm{e}^{\gamma\left(t_{0}-t\right)},
$$

where $\Phi^{B}=\gamma^{-1} f^{B}$. If $\Phi^{B} \in B$ then $x=\Phi^{B}$ is an asymptotically stable stationary point of (2).

Proof In the box $B,(2)$ reduces to the linear system $x^{\prime}=f^{B}-\gamma x$. Thus, the solutions are given by (5). When $t \rightarrow+\infty, x(t) \rightarrow \Phi^{B}$, until $x(t)$ encounters the boundary of the box $B$. When $\Phi^{B} \in B$, then $x=\Phi^{B}$ is a stable node of (2).

The solutions given by (5) are curves originated at $x\left(t_{0}\right)$ and converging toward $\Phi^{B}$, called the focal point of box $B[1,2]$. We only consider throughout the paper the generic case where the focal point $\Phi^{B}$ does not lie on the threshold hyperplanes which constitute the boundary of $B$. The face separating two adjacent $n$-boxes will be called a $(n-1)$-box or a wall.

Theorem 2 If $f_{i}^{B}<\gamma_{i} \theta_{i}^{q_{i}}$, all trajectories in $B$ that are encountering the wall $x_{i}=\theta_{i}^{q_{i}}$ are leaving the box $B$. Similarly, if $f_{i}^{B}>\gamma_{i} \theta_{i}^{q_{i}+1}$, all trajectories in $B$ that are encountering the wall $x_{i}=\theta_{i}^{q_{i}+1}$ are leaving the box $B$.

Proof Suppose that $f_{i}^{B}<\gamma_{i} \theta_{i}^{q_{i}}$ and the trajectory $x(t)$ belongs to the wall $x_{i}=\theta_{i}^{q_{i}}$ at time $t_{0}$. Then $x_{i}\left(t_{0}\right)-\Phi_{i}^{B}>0$ and, according to (5), the component $x_{i}(t)$ of $x(t)$ is decreasing. Thus $x_{i}(t)<\theta_{i}^{q_{i}}$ for $t>t_{0}$, that is, the solution $x(t)$ leaves the box. The proof is similar in the other case.

If $\theta_{i}^{q_{i}}<f_{i}^{B} / \gamma_{i}<\theta_{i}^{q_{i}+1}$, all trajectories in $B$ that are encountering the walls $x_{i}=\theta_{i}^{q_{i}}$ or $x_{i}=\theta_{i}^{q_{i}+1}$ enter the box $B$ from these walls. However, the trajectories can leave the box $B$ from another wall.

Following Plahte et al. [7], a wall separating two boxes will be called a black wall (resp. a white wall) if trajectories leave (resp. enter) both boxes from the wall. It will be called a transparent wall if trajectories enter one box from the wall and leave the other box.

One can construct the state transition graph $[1,2]$ for system $(2)$, which is a directed graph representing the passage of the trajectories from one box to the adjacent boxes. To each box is associated a vertex. To each wall $W$ separating two adjacent boxes $B_{1}$ and $B_{2}$ is associated a directed edge connecting the vertices corresponding to the boxes $B_{1}$ and $B_{2}$.

INRIA 
If $W$ is black, then there is two edges between $B_{1}$ and $B_{2}$ in the transition graph. If $W$ is transparent (resp. white), then there is one edge (resp. no edge) between $B_{1}$ and $B_{2}$ in the transition graph (Figs. 3, 4, and 5).

If $W$ is transparent, trajectories in $B_{1}$ and $B_{2}$ can be joined in a single continuous trajectory satisfying the PL system. In the other case, when $W$ is black or white, there is no indication of how a solution can be continued. If $W$ is black, then near the wall all the solutions are approaching it from both sides as $t$ increases, and none of them can leave $W$. A solution which passes through a point of the wall $W$ at $t=t_{0}$ will therefore remain on $W$ for $t>t_{0}$ until it reaches the boundary of $W$. If $W$ is white, then a solution which passes through a point of the wall $W$ at $t=t_{0}$ may either go off the wall $W$ into the box $B_{1}$ or $B_{2}$, or remain on $W$ for $t>t_{0}$. In the later case the solution may go off $W$ at any moment. On white wall the motion is therefore unstable.

\section{Filippov solutions of the PL system}

In this section we describe the classical concept of Filippov solutions in two cases, describing a motion along a wall (first case) or along the intersection of two walls (second case).

To provide the existence and the possibility for solutions to be continued on both black or white walls, it is necessary to define the right-hand side of system (1) at these point of discontinuity. The simplest way to achieve this is the convex definition of Filippov [3]. Let $x \in W$, where $W$ is a black (or white) wall separating the boxes $B_{1}$ and $B_{2}$. Consider the linear segment joining the endpoints of the vectors $f^{B_{1}}-\gamma x$ and $f^{B_{2}}-\gamma x$. This segment intersect the hyperplane containing the wall $W$ (Fig. 1), the intersection is the end point of the vector $f^{W}-\gamma x$ which determines the velocity of motion

$$
x^{\prime}=f^{W}-\gamma x
$$

on the wall $W$. Such a solution is called a sliding motion.

On each black or white wall $W$ lying in the hyperplane $C_{i}^{j}=\left\{x \in C: x_{i}=\theta_{i}^{j}\right\}$, equation (6) defines a sliding motion. Since the constant vector $f^{W}$ in (6) depends on $W$, the resulting system on $C_{i}^{j}$ is a $(n-1)$-dimensional PL system of the form

$$
x^{\prime}=g_{i}^{j}(x)-\gamma x, \quad x \in C_{i}^{j}, \quad g_{i}^{j}: C_{i}^{j} \rightarrow \mathbb{R}^{n-1},
$$

where the mapping $g_{i}^{j}$ is piecewise constant. This PL system is similar to system (2), and can be studied by the same methods, however it is only defined on black or white walls. The threshold hyperplanes $x_{i^{\prime}}=\theta_{i^{\prime}}^{j^{\prime}}$, where $i^{\prime} \neq i$ and $j^{\prime}=1, \cdots, N_{i^{\prime}}$ separate the cone $C_{i}^{j}$ into $N /\left(N_{i}+1\right)$ boxes of dimension $n-1$. These $(n-1)$-boxes are the walls separating adjacent $n$-boxes of system (2). The solutions of (6) are curves converging toward the focal point $\Phi^{W}=\gamma^{-1} f^{W}$. 
Theorem 3 Assume that $W$ is in the hyperplane $C_{i}^{j}$ and $W$ is black or white, that is, $\left(f_{i}^{B_{2}}-\gamma_{i} \theta_{i}^{j}\right)\left(f_{i}^{B_{1}}-\gamma_{i} \theta_{i}^{j}\right)<0$. The constant vector $f^{W}$ in (6) is given by

$$
f^{W}=\alpha f^{B_{1}}+(1-\alpha) f^{B_{2}}, \quad \alpha=\frac{f_{i}^{B_{2}}-\gamma_{i} \theta_{i}^{j}}{f_{i}^{B_{2}}-f_{i}^{B_{1}}} .
$$

The focal point $\Phi^{W}$ is the intersection of the linear segment $\Phi^{B_{1}} \Phi^{B_{2}}$ with the hyperplane $C_{i}^{j}$ :

$$
\Phi^{W}=C_{i}^{j} \cap \operatorname{conv}\left(\Phi^{B_{1}}, \Phi^{B_{2}}\right) .
$$

Proof The segment joining the endpoints of the vectors $f^{B_{1}}-\gamma x$ and $f^{B_{2}}-\gamma x$ is expressed by

$$
\alpha f^{B_{1}}+(1-\alpha) f^{B_{2}}-\gamma x, \quad 0 \leq \alpha \leq 1 .
$$

The value of $\alpha$ in (7) is found from de condition $\alpha f_{i}^{B_{1}}+(1-\alpha) f_{i}^{B_{2}}-\gamma_{i} \theta_{i}^{j}=0$ which means that the vector $f^{W}-\gamma x$ is in the hyperplane $x_{i}=\theta_{i}^{j}$. Since $\left(f_{i}^{B_{2}}-\gamma_{i} \theta_{i}^{j}\right)\left(f_{i}^{B_{1}}-\gamma_{i} \theta_{i}^{j}\right)<0$, one has that $0<\alpha<1$. From (7) one has that

$$
\Phi^{W}=\alpha \Phi^{B_{1}}+(1-\alpha) \Phi^{B_{2}}, \quad 0<\alpha<1 .
$$

Thus, $\Phi^{W}$ is the intersection of the linear segment $\Phi^{B_{1}} \Phi^{B_{2}}$ with the hyperplane $C_{i}^{j}$.

Let us now describe the velocity of motion along a $(n-2)$-box $P$ which is the intersection of two threshold hyperplanes. This box is the common face of four $n$-boxes $B_{1}, B_{2}, B_{3}$ and $B_{4}$ (Fig. 2). Let $x \in P$. Following [3], we consider the smallest convex set

$$
K(x)=\operatorname{conv}\left(f^{B_{1}}-\gamma x, f^{B_{2}}-\gamma x, f^{B_{3}}-\gamma x, f^{B_{4}}-\gamma x\right)
$$

containing the vectors $f^{B_{i}}-\gamma x, i=1$ to 4 . In general the intersection of $K(x)$ and the $(n-2)$-hyperplane containing $P$ does not consist of only one point. In generic cases, this intersection is a linear segment or is empty. If it is empty then there are no solutions lying on $P$. If it is nonempty then one obtains a differential inclusion

$$
x^{\prime}(t) \in f^{P}-\gamma x(t),
$$

which determines the velocity of motion along $P$.

Theorem 4 Assume that $P$ is in the $(n-2)$-dimensional hyperplane $C_{i k}^{j l}=\left\{x \in C: x_{i}=\right.$ $\left.\theta_{i}^{j}, x_{k}=\theta_{k}^{l}\right\}$. The set $f^{P}$ in (8) consists of all points $\alpha_{1} f^{B_{1}}+\alpha_{2} f^{B_{2}}+\alpha_{3} f^{B_{3}}+\alpha_{4} f^{B_{4}}$, where the $\alpha_{i}$ are the positive solutions of the linear system

$$
\begin{aligned}
& \alpha_{1}+\alpha_{2}+\alpha_{3}+\alpha_{4}=1, \\
& \alpha_{1} f_{i}^{B_{1}}+\alpha_{2} f_{i}^{B_{2}}+\alpha_{3} f_{i}^{B_{3}}+\alpha_{4} f_{i}^{B_{4}}=\gamma_{i} \theta_{i}^{j}, \\
& \alpha_{1} f_{k}^{B_{1}}+\alpha_{2} f_{k}^{B_{2}}+\alpha_{3} f_{k}^{B_{3}}+\alpha_{4} f_{k}^{B_{4}}=\gamma_{k} \theta_{k}^{l} .
\end{aligned}
$$

The focal set $\Phi^{P}=\gamma^{-1} f^{P}$ is the intersection of the smallest convex containing the focal points $\Phi^{B_{1}}, \Phi^{B_{2}}, \Phi^{B_{3}}$ and $\Phi^{B_{4}}$, with the hyperplane $C_{i k}^{j l}$ :

$$
\Phi^{P}=C_{i k}^{j l} \cap \operatorname{conv}\left(\Phi^{B_{1}}, \Phi^{B_{2}}, \Phi^{B_{3}}, \Phi^{B_{4}}\right) .
$$


Proof For $x \in P$, the smallest convex set $K(x)$ containing the vectors $f^{B_{i}}-\gamma x, i=1$ to 4 , is the set of all vectors of the form

$$
\alpha_{1} f^{B_{1}}+\alpha_{2} f^{B_{2}}+\alpha_{3} f^{B_{3}}+\alpha_{4} f^{B_{4}}-\gamma x, \quad \alpha_{i} \geq 0, \quad \sum \alpha_{i}=1
$$

This vector belongs to the hyperplane $C_{i j}^{k l}$ if and only if the $\alpha_{i}$ are the positive solutions of the linear system (9). From (10) one has that

$$
\Phi^{P}=\alpha_{1} \Phi^{B_{1}}+\alpha_{2} \Phi^{B_{2}}+\alpha_{3} \Phi^{B_{3}}+\alpha_{4} \Phi^{B_{4}}, \quad \alpha_{i} \geq 0, \quad \sum \alpha_{i}=1 .
$$

Thus, $\Phi^{P}$ is the intersection of $C_{i k}^{j l}$ with $\operatorname{conv}\left(\Phi^{B_{1}}, \Phi^{B_{2}}, \Phi^{B_{3}}, \Phi^{B_{4}}\right)$.

The differential inclusion means that the motion is not determined in a unique way by this concept of Filippov solutions. To choose between theses different trajectories, we would need a more specific concept of solutions.

This construction may be continued on all intersection of threshold planes. It will be achieved in Section 5 on some illustrative examples.

\section{Regular and singular stationary points}

We show now the utility of this concept of solutions in the study of the singular steady states.

Snoussi and Thomas [10] introduced the concepts of regular stationary points (RSP) and singular stationary points (SSP) in models comprising step functions. A RSP is a stationary point where none of the variables lie on their thresholds. A SSP is a stationary point where one or more variables lie on their thresholds. The RSP are simply defined by putting $x^{\prime}=0$ in (2), that is, by equation $\gamma x=f(x)$. This equation has a solution if an only if for some box $B$, the focal point $\Phi^{B}$ belongs to $B$. According to Theorem $1, x=\Phi^{B}$ is an asymptotically stable RSP. Since the PL system is not defined on the threshold planes, SSP cannot be defined by $x^{\prime}=0$. Their definition is not so obvious (see [10], p. 984).

In the present paper, we used the concept of solutions of (2) in the sense of Filippov, so we have no problem with the definition of stationary points. Indeed the point $x=c$ is called a stationary point for the PL system (2) if it is a solution, that is, if $x(t) \equiv c$ is a solution (in the sense of Filippov) of (2). A SSP is a stationary point which belongs to some threshold plane.

Theorem 5 If a SSP belongs to a black wall, then it is an asymptotically stable solution of (2).

Proof Let $x=p$ be a SSP which belongs to the black wall $W$. Then, one has $p=\Phi^{W} \in W$. Thus $\Phi^{W}$ is a stable node of (6) and hence, an asymptotically stable solution of (2).

To avoid the difficulties of [10] in the definition of SSP, Plahte et al. [7, 8] suggested to define a stationary point of the PL system as the limit of stationary points of homologous 
continuous systems, when the steepness of the sigmoid functions increases. The stationary point is said to be singular if this limit lies in some threshold plane. With this definition, it is not so obvious to handle the concept of SSP at the limit. For instance, these authors wrote that "Asymptotically stable steady point of the PL system can only be located in transparent walls. If a PL trajectory encounters a black wall, it simply come to a dead stop in a stable, but not asymptotically stable singular point."(see [7], p. 284). This claim is contradicted by the previous theorem (cf. the first example of the next section).

The analysis of SSP lying on a $(n-2)$-box $P$ which is the intersection of two threshold planes is more delicate. Let $p \in P$ be a stationary point, then $x(t) \equiv p$ is a solution of the differential inclusion (8). Thus $p \in f^{P}$ and $f^{P} \cap P \neq \emptyset$. If $f^{P}$ does not consist of only point $p$, then $p$ is unstable. If $f^{P}=\{p\}$, then $x=f^{P}$ is an asymptotically stable stationary point of (8). Hence it is a stationary point of (2). Further analyses are needed to study the stability. We can study in the same manner the SSP which belong to the intersection of more than two threshold hyperplanes.

\section{Examples}

This section is intended to illustrate the applicability of our approach in some typical examples which appeared in the literature.

In the following examples, we consider the form (3) of the PL system. If there is only one threshold value $\theta_{i}^{1}$ for the variable $x_{i}$, we denote it simply $\theta_{i}$ and we denote $z_{i}=S\left(x_{i}, \theta_{i}\right)$. Whenever necessary, we specify $z_{i}^{j}$ as $z_{i}^{j+}$ and we denote $z_{i}^{j-}=1-z_{i}^{j+}$.

\subsection{Example of Plahte, Mestl and Omholt [7]}

In this first example we have the case of an asymptotically stable SSP in a black wall; we obtain the unstability of the other SSP.

Consider a two dimensional system with one threshold value $\theta_{i}$ for each variable $x_{i}$. The equations are

$$
\begin{aligned}
& x_{1}^{\prime}=k_{1}\left(z_{1}^{+} z_{2}^{-}+z_{1}^{-} z_{2}^{+}\right)-x_{1}, \\
& x_{2}^{\prime}=k_{2} z_{1}^{-}-x_{2} .
\end{aligned}
$$

The positive cone is separated in four boxes $B^{00}, B^{01}, B^{10}$, and $B^{11}$ (Fig. 3). Assume that $k_{1}>\theta_{1}$ and $k_{2}>\theta_{2}$. The focal points are $f^{00}=\left(0, k_{2}\right) \in B^{01}, f^{01}=\left(k_{1}, k_{2}\right) \in B^{11}$, $f^{10}=\left(k_{1}, 0\right) \in B^{10}$ and $f^{11}=(0,0) \in B^{00}$. Thus, $f^{10}$ is an asymptotically stable RSP. The wall $W_{1}$ is white, $W_{2}$ is black, $W_{3}$ and $W_{4}$ are transparent. The sliding motion on $W_{1}$ is given by $x_{2}^{\prime}=k_{2}-\theta_{1} k_{2} / k_{1}-x_{2}$. The sliding motion on $W_{2}$ is given by $x_{2}^{\prime}=\theta_{1} k_{2} / k_{1}-x_{2}$. Assume that $k_{1}<2 \theta_{1}$ and $k_{2} / k_{1}>\theta_{2} / \theta_{1}$. Then, the focal point $f^{W_{1}}=\left(\theta_{1}, k_{2}-\theta_{1} k_{2} / k_{1}\right)$ belongs to $W_{1}$ and the focal point $f^{W_{2}}=\left(\theta_{1}, \theta_{1} k_{2} / k_{1}\right)$ belongs to $W_{2}$. Thus $f^{W_{1}}$ is is an unstable SSP and $f^{W_{2}}$ is an asymptotically stable SSP. Moreover, the point $P=\left(\theta_{1}, \theta_{2}\right)$ belongs to the convex set $\operatorname{conv}\left(f^{00}, f^{01}, f^{10}, f^{11}\right)$, thus $P$ is a SSP; it is unstable. 


\subsection{Example of Snoussi and Thomas [10]}

Here we obtain the location (and not only the detection) of the SSP. This example was proposed in [10] as an illustration of the method of detection of SSP. Consider a two dimensional system with two threshold values $\theta_{i}^{1}<\theta_{i}^{2}$ for each variable $x_{i}$. The equations are

$$
\begin{aligned}
& x_{1}^{\prime}=k_{1} z_{1}^{1}+k_{3} z_{2}^{2}-x_{1}, \\
& x_{2}^{\prime}=k_{2} z_{1}^{2}+k_{4} z_{2}^{1}-x_{2} .
\end{aligned}
$$

The positive cone is separated in nine boxes $B^{i j}, i, j=0,1,2$ (Fig. 4). Assume that $\theta_{1}^{1}<k_{1}<\theta_{1}^{2}, \theta_{1}^{1}<k_{3}<\theta_{1}^{2}<k_{1}+k_{3}$, and $\theta_{2}^{1}<k_{2}<\theta_{2}^{2}, \theta_{2}^{1}<k_{4}<\theta_{2}^{2}<k_{2}+k_{4}$. The focal points are $f^{00}=(0,0) \in B^{00}, f^{10}=\left(k_{1}, 0\right) \in B^{10}, f^{20}=\left(k_{1}, k_{2}\right) \in B^{11}$, $f^{01}=\left(0, k_{4}\right) \in B^{01}, f^{11}=\left(k_{1}, k_{4}\right) \in B^{11}, f^{21}=\left(k_{1}, k_{2}+k_{4}\right) \in B^{12}, f^{02}=\left(k_{3}, k_{4}\right) \in B^{11}$, $f^{12}=\left(k_{1}+k_{3}, k_{4}\right) \in B^{21}$ and $f^{22}=\left(k_{1}+k_{3}, k_{2}+k_{4}\right) \in B^{22}$. Thus, $f^{00}, f^{10}, f^{01}, f^{11}$ and $f^{22}$ are asymptotically stable RSP. Let us determine the SSP. A wall $W$ separating the boxes $B^{i j}$ and $B^{k l}$ is denoted by $W=\left(B^{i j}, B^{k l}\right)$. The walls $W_{1}=\left(B^{00}, B^{01}\right), W_{2}=\left(B^{00}, B^{10}\right)$, $W_{3}=\left(B^{10}, B^{11}\right)$ and $W_{4}=\left(W^{01}, W^{11}\right)$ are white. All other walls are transparent. The sliding motion on $W_{1}$ is given by $x_{1}^{\prime}=-x_{1}$; its focal point is $f^{W_{1}}=\left(0, \theta_{2}^{1}\right) \in W_{1}$. The sliding motion on $W_{2}$ is given by $x_{2}^{\prime}=-x_{2}$; its focal point is $f^{W_{2}}=\left(\theta_{1}^{1}, 0\right) \in W_{2}$. The sliding motion on $W_{3}$ is given by $x_{1}^{\prime}=k_{1}-x_{1}$; its focal point is $f^{W_{3}}=\left(k_{1}, \theta_{2}^{1}\right) \in W_{3}$. The sliding motion on $W_{4}$ is given by $x_{2}^{\prime}=k_{4}-x_{2}$; its focal point is $f^{W_{4}}=\left(\theta_{1}^{1}, k_{4}\right) \in W_{4}$. Thus $f^{W_{1}}, f^{W_{2}}, f^{W_{3}}$ and $f^{W_{4}}$ are SSP; they are unstable. Consider now the intersections of the threshold planes. One has

$$
\left(\theta_{1}^{1}, \theta_{2}^{1}\right) \in \operatorname{conv}\left(f^{00}, f^{01}, f^{10}, f^{11}\right), \quad\left(\theta_{1}^{2}, \theta_{2}^{2}\right) \in \operatorname{conv}\left(f^{11}, f^{21}, f^{12}, f^{22}\right) .
$$

Thus $P_{1}=\left(\theta_{1}^{1}, \theta_{2}^{1}\right)$ and $P_{2}=\left(\theta_{1}^{2}, \theta_{2}^{2}\right)$ are SSP; they are unstable. Moreover

$$
\left(\theta_{1}^{1}, \theta_{2}^{2}\right) \notin \operatorname{conv}\left(f^{01}, f^{11}, f^{12}, f^{02}\right), \quad\left(\theta_{1}^{2}, \theta_{2}^{1}\right) \notin \operatorname{conv}\left(f^{10}, f^{20}, f^{21}, f^{11}\right) .
$$

Thus, $P_{3}=\left(\theta_{1}^{1}, \theta_{2}^{2}\right)$ and $P_{4}=\left(\theta_{1}^{2}, \theta_{2}^{1}\right)$ are not stationary points. Hence (12) has eleven stationary points, five of them are regular, 6 are singular. Notice that in our approach, the SSP belonging to white walls are not only detected but also located (compare with [10], p. 981-982).

\subsection{Example of Thomas [7]}

We consider a gene regulatory model investigated by Thomas ([11]) and studied by Plahte et al. ([7]). These authors use for their study a continuous approximation of the system by logoids. We recall briefly their approach and results afterwards. We obtain a complete analysis of the example.

Consider the three dimensional system with one threshold value $\theta_{i}$ for each variable $x_{i}$.

$$
\begin{aligned}
& x_{1}^{\prime}=k_{1} z_{2}^{-} z_{3}^{+}-\gamma_{1} x_{1} \\
& x_{2}^{\prime}=k_{2} z_{1}^{+} z_{3}^{+}-\gamma_{2} x_{2} \\
& x_{3}^{\prime}=k_{3}\left(z_{1}^{-}+z_{2}^{+}-z_{1}^{-} z_{2}^{+}\right)-\gamma_{3} x_{3} .
\end{aligned}
$$


The positive cone is separated in eight boxes $B^{i j k}, i, j, k=0,1$. Assume that $0<g_{i}<1$, where $g_{i}=\gamma_{i} \theta_{i} / k_{i}, i=1,2,3$. The focal points are $\Phi^{000}=\Phi^{010}=\Phi^{011}=\Phi^{110}=$ $\left(0,0, k_{3} / \gamma_{3}\right) \in B^{001}, \Phi^{100}=(0,0,0) \in B^{000}, \Phi^{001}=\left(k_{1} / \gamma_{1}, 0, k_{3} / \gamma_{3}\right) \in B^{101}, \Phi^{101}=$ $\left(k_{1} / \gamma_{1}, k_{2} / \gamma_{2}, 0\right) \in B^{110}$, and finally $\Phi^{111}=\left(0, k_{2} / \gamma_{2}, k_{3} / \gamma_{3}\right) \in B^{011}$. Thus, (13) has no RSP. Let us determine the SSP. All walls are transparent. Hence there is no sliding motion on the walls. The SSP must lie on the intersection of two or more threshold planes. Let $C_{i j}=\left\{x \in C: x_{i}=\theta_{i}, x_{j}=\theta_{j}\right\}$. Let $P_{1}=\left\{x \in C_{23}: x_{1}>\theta_{1}\right\} P_{2}=\left\{x \in C_{13}: 0 \leq x_{2}<\right.$ $\left.\theta_{2}\right\}$ and $P_{3}=\left\{x \in C_{12}: x_{3}>\theta_{3}\right\}$. The sliding motion on $P_{i}$ is given by the differential inclusion

$$
x_{i}^{\prime}(t) \in k_{i} A_{i}-\gamma_{i} x_{i}(t), \quad x_{i}(t) \in P_{i}
$$

where

$$
\begin{gathered}
A_{1}=\left[\min \left(0, g_{2}-g_{3}\right), \max \left(g_{2}, 1-g_{3}\right)\right], \\
A_{2}=\left[\min \left(0, g_{1}-g_{3}\right), \max \left(g_{1}, 1-g_{3}\right)\right], \\
A_{3}=\left[\min \left(1-g_{1}, 1-g_{2}\right), \max \left(1,2-g_{1}-g_{2}\right)\right] .
\end{gathered}
$$

One has a SSP on $P_{i}$ if and only if the corresponding focal set intersects $P_{i}$, that is,

$$
k_{i} A_{i} / \gamma_{i} \cap P_{i} \neq \emptyset
$$

For $i=1$, condition (14) is equivalent to $g_{1}<\max \left(g_{2}, 1-g_{3}\right)$. For $i=2$, condition (14) is equivalent to $g_{3}>g_{1}-g_{2}$. For $i=3$, condition (14) is equivalent to $g_{3}<2-g_{1}-g_{2}$. Hence the cube $0<g_{i}<1, i=1,2,3$ in parameter space, is separated into four different regions, in which one, two or three SSP may exist on $P_{i}, i=1,2,3$ (Fig. 6).

The line $P_{2}^{\prime}=\left\{x \in C_{13}: x_{2}>\theta_{2}\right\}$ is the common edge of the boxes $B^{010}, B^{110}, B^{011}$ and $B^{111}$. One has $C_{13} \cap \operatorname{conv}\left(\Phi^{010}, \Phi^{110}, \Phi^{011}, \Phi^{111}\right)=\emptyset$. Hence, there is no sliding motion on $P_{2}^{\prime}$. Also, one has

$$
C_{12} \cap \operatorname{conv}\left(\Phi^{000}, \Phi^{100}, \Phi^{010}, \Phi^{110}\right)=\emptyset, \quad C_{23} \cap \operatorname{conv}\left(\Phi^{000}, \Phi^{010}, \Phi^{011}, \Phi^{001}\right)=\emptyset .
$$

Thus, there is no sliding motions on the segments $P_{3}^{\prime}=\left\{x \in C_{12}: 0 \leq x_{3}<\theta_{3}\right\}$ and $P_{1}^{\prime}=\left\{x \in C_{23}: 0 \leq x_{1}<\theta_{1}\right\}$.

Let us consider now the intersection of the three threshold planes. The point $P=$ $\left(\theta_{1}, \theta_{2}, \theta_{3}\right)$ is a SSP if and only if it belongs to the convex set containing all focal points $\Phi^{i j k}$, $i, j, k=0,1$. This case arises if and only if $g_{1}+g_{3}>g_{2}, g_{2}+g_{3}>g_{1}$ and $g_{1}+g_{2}+g_{3}<2$ (see Fig. 6 which represent the domain in parameter space in which $P$ is a SSP).

Let us consider now the continuous approach of [7]. When the step functions $z(x)$ in (3) are replaced by continuous approximations $Z(x, \delta)$, the resulting system

$$
x^{\prime}=F(Z(x, \delta))-\gamma x,
$$

is a continuous analogue of (3). Here

$$
Z(x, \delta)=\left(Z_{1}^{1}, \cdots, Z_{1}^{N_{1}}, \cdots, Z_{n}^{1}, \cdots, Z_{n}^{N_{n}}\right), \quad Z_{i}^{j}=\Sigma\left(x_{i}, \theta_{i}^{j}, \delta\right),
$$


and $\Sigma(\xi, \theta, \delta)$ is a sigmoid function, such that the steepness increases when $\delta \rightarrow 0$, that is,

$$
\lim _{\delta \rightarrow 0} \Sigma(\xi, \theta, \delta)=S(\xi, \theta) .
$$

Plahte et al. [7] considered the SSP of (13) as the limits of the corresponding continuous analogue

$$
\begin{aligned}
& x_{1}^{\prime}=k_{1} Z_{2}^{-} Z_{3}^{+}-\gamma_{1} x_{1}, \\
& x_{2}^{\prime}=k_{2} Z_{1}^{+} Z_{3}^{+}-\gamma_{2} x_{2}, \\
& x_{3}^{\prime}=k_{3}\left(Z_{1}^{-}+Z_{2}^{+}-Z_{1}^{-} Z_{2}^{+}\right)-\gamma_{3} x_{3} .
\end{aligned}
$$

of (13).

They detected a steady state $\left(x_{1}^{2}(\delta), x_{2}^{2}(\delta), x_{3}^{2}(\delta)\right)$ satisfying

$$
\lim _{\delta \rightarrow 0} x_{1}^{2}(\delta)=\theta_{1}, \quad \lim _{\delta \rightarrow 0} x_{2}^{2}(\delta)=k_{2}\left(1-g_{3}\right) g_{1} / \gamma_{2}, \quad \lim _{\delta \rightarrow 0} x_{3}^{2}(\delta)=\theta_{3},
$$

This limit belongs to $P_{2}$ if and only if $g_{3}>1-g_{2} / g_{1}$. They also detected a steady state $\left(x_{1}^{3}(\delta), x_{2}^{3}(\delta), x_{3}^{3}(\delta)\right)$ satisfying

$$
\lim _{\delta \rightarrow 0} x_{1}^{3}(\delta)=\theta_{1}, \quad \lim _{\delta \rightarrow 0} x_{2}^{3}(\delta)=\theta_{2}, \quad \lim _{\delta \rightarrow 0} x_{3}^{3}(\delta)=k_{3}\left(1-g_{1} g_{2}\right) / \gamma_{3} .
$$

This limit belongs to $P_{3}$ if and only if $g_{3}<1-g_{1} g_{2}$. Notice that the domains of existence in parameter space of these two SSP are included in our domains (Fig. 6).

It is claimed in [7] that steady points may only exist on $P_{2}$ and $P_{3}$ which correspond to circuits in the transition graph (Fig. 5). In fact, it is easily seen that (16) also has a third steady state $\left(x_{1}^{1}(\delta), x_{2}^{1}(\delta), x_{3}^{1}(\delta)\right)$ satisfying

$$
\lim _{\delta \rightarrow 0} x_{1}^{1}(\delta)=k_{1}\left(1-g_{3}\right) g_{2} / \gamma_{1}, \quad \lim _{\delta \rightarrow 0} x_{2}^{1}(\delta)=\theta_{2}, \quad \lim _{\delta \rightarrow 0} x_{3}^{1}(\delta)=\theta_{3} .
$$

This limit belongs to $P_{1}$ if and only if $g_{3}<1-g_{1} / g_{2}$ and the corresponding domain of existence is included in our domain (Fig. 6). Another continuous approximation of the system (13) would have give SSP localised elsewhere, but always in the focal sets given by our analysis of the PL system.

\section{Conclusion}

The concept of Filippov solutions enables a rigorous and clear treatment of the problem of singular solutions. It can provide new tools for exploring the interesting problems arising from these threshold biological models. Of course, the problem of the ambiguity of the dynamical behaviour in the case of the differential inclusion (8) stays open: it should be solved by some method of regularization ([12]), either by continuous homologous systems, or by the way of a stochastic process.

Acknowledgements: The authors thank the GdR CNRS Automatique and the French COREV group for their support.

$\mathrm{RR} \mathrm{n}^{\circ} 4207$ 


\section{References}

[1] L. Glass, J. Pasternack, Prediction of limit cycles in mathematical models of biological oscillations, Bulletin of Mathematical Biology, Vol. 40 (1978), 27-44.

[2] L. Glass, J. Pasternack, Stable oscillations in mathematical models of biological control systems, J. Math. Biology, Vol. 6 (1978), 207-223.

[3] A. F. Filippov, Differential Equations with Discontinuous Righthand Sides, Kluwer Academic Publishers (1988)

[4] H. de Jong, Modeling and simulation of Genetic regulatory systems: a literature review, Research report no. 4032, September 2000, INRIA, http://www.inria.fr/rrrt/rr4032.html.

[5] S. A. Kauffman, J. Urbain, R. Thomas, Towards a logical analysis of the immune response, Journal of Theoretical Biology, Vol. 114 (1985), 527-561.

[6] T. Mestl, E. Plahte, S. W. Omholt, Periodic solutions in systems of piecewiselinear differential equations, Dynamics and Stability of Systems, Vol. 10 (1995), 179193.

[7] E. Plahte, T. Mestl, S. W. Omholt, Global analysis of steady points for systems of differential equations with sigmoid interactions, Dynamics and Stability of Systems, Vol. 9 (1994), 275-291.

[8] E. Plahte, T. Mestl, S. W. Omholt, A methodological basis for description and analysis of systems with complex switch-like interaction, J. Math. Biol., Vol. 36 (1998), 321-348.

[9] E. H. Snoussi, Qualitative dynamics of piecewise-linear differential equations : a discrete mapping approach, Dynamics and Stability of Systems, Vol. 4 (1989), 189207.

[10] E. H. Snoussi, R. Thomas, Logical identification of all steady states : the concept of feedback loop characteristic states Bulletin of Mathematical Biology, Vol. 55 (1993), 973-991.

[11] R. Thomas, Kinetics logic : a Boolean analysis of the dynamic behavior of control circuits, Lecture Notes in Biomathematics 29, Springer (1979)

[12] V.I. Utkin, Sliding modes in control and optimization Communications and Control Engineering Series, Springer (1992) 


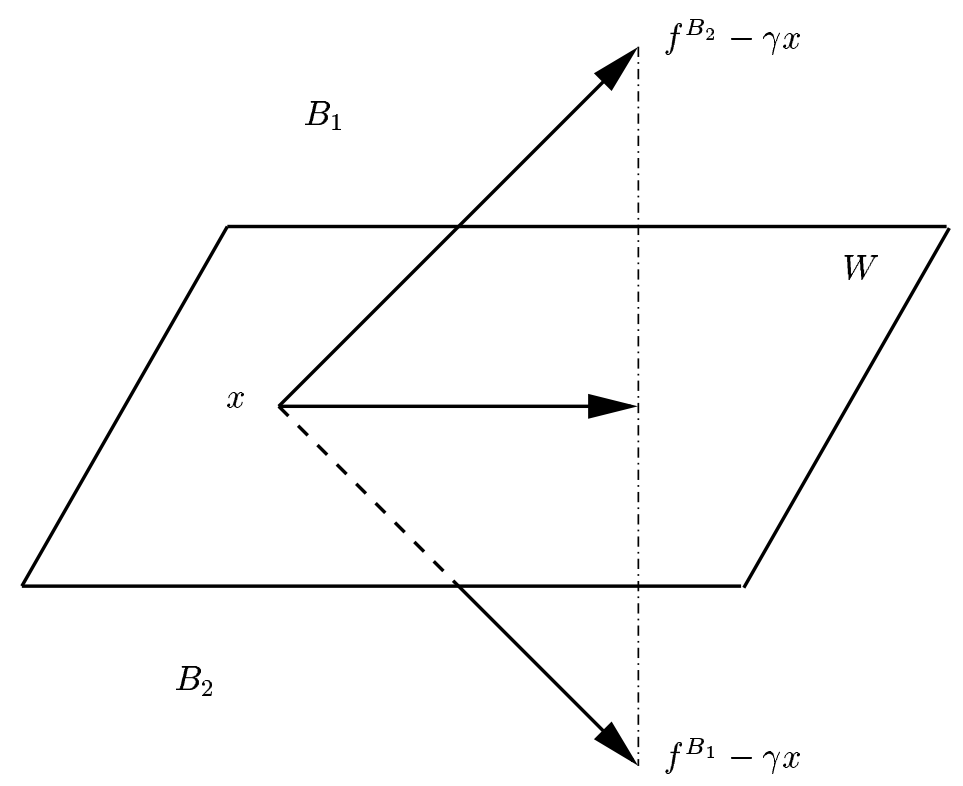

Figure 1: The velocity of motion along a threshold hyperplane.

$\mathrm{RR} \mathrm{n}^{\circ} 4207$ 


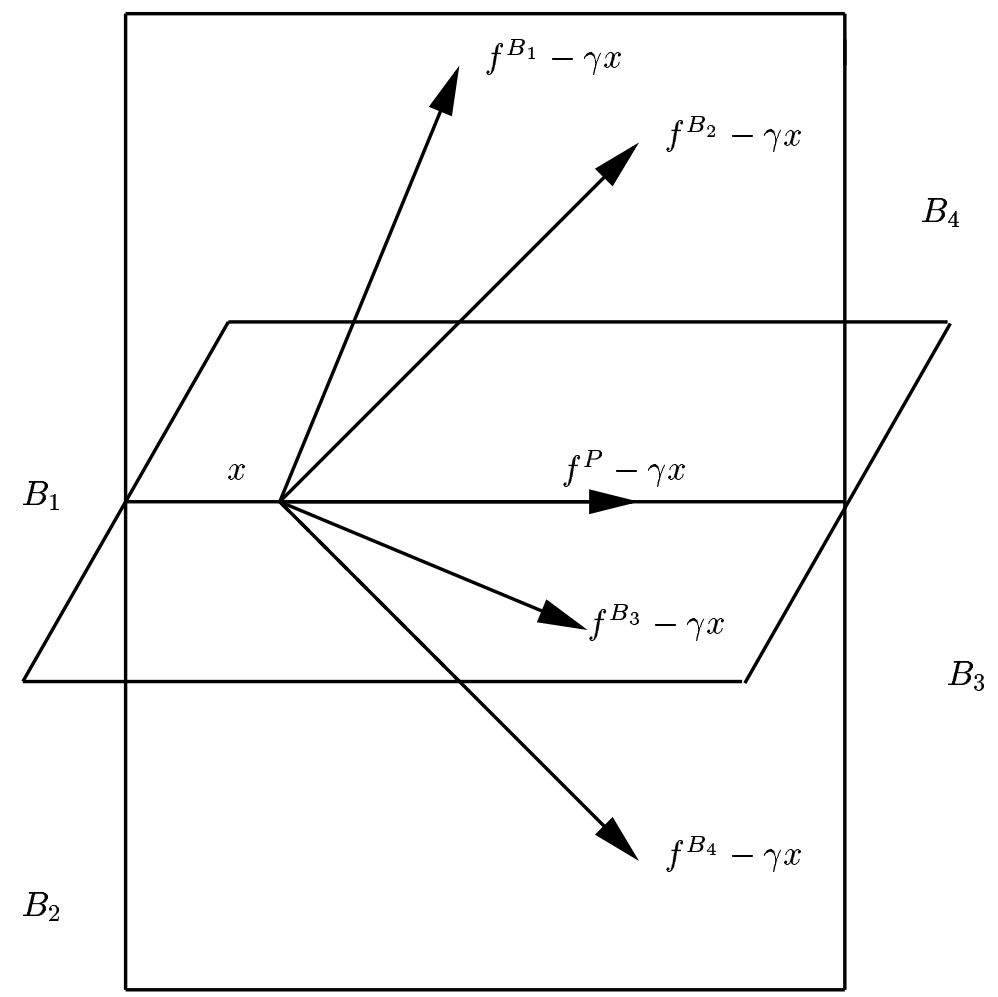

Figure 2: The velocity of motion along the intersection of two threshold hyperplanes. 


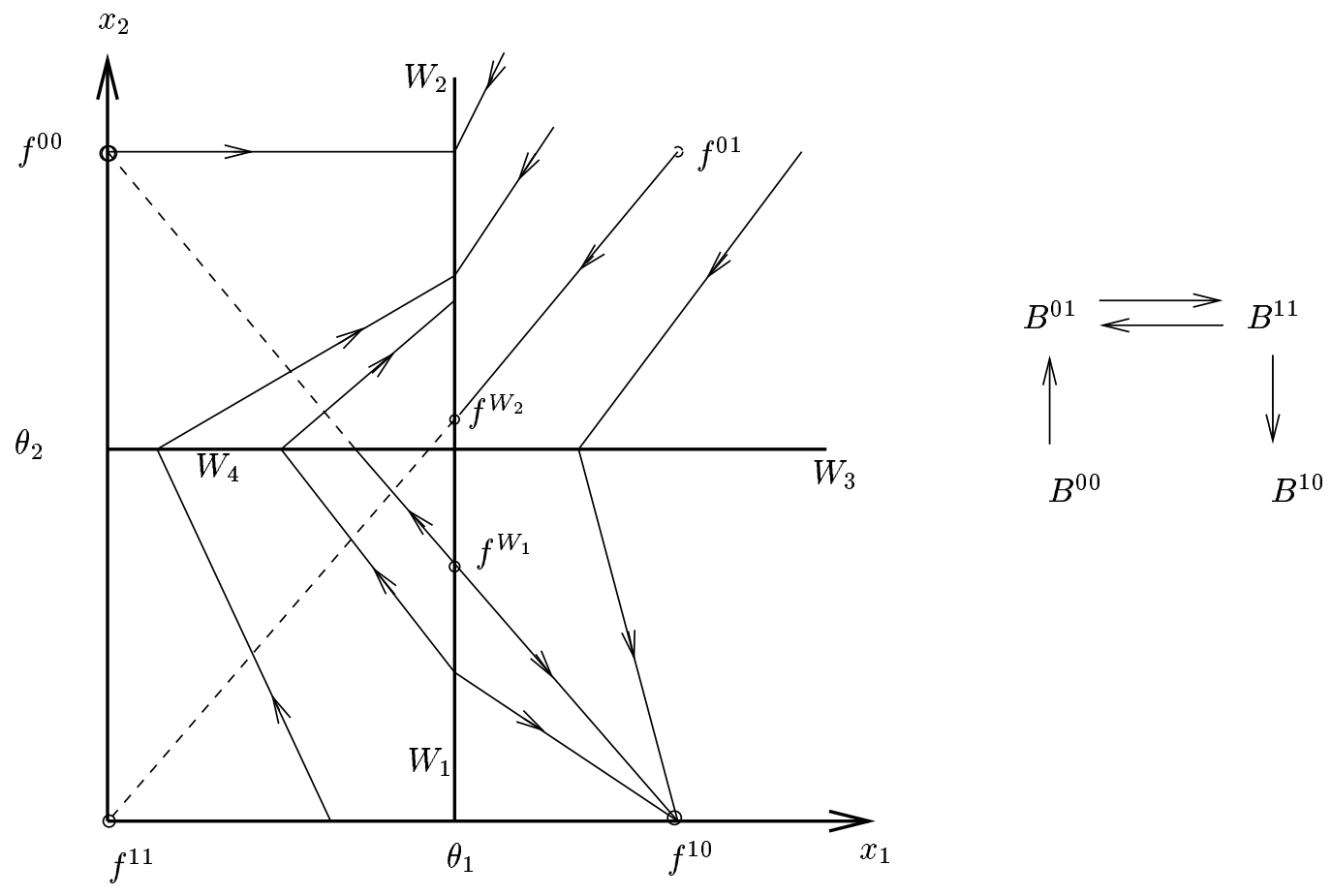

Figure 3: The flow and transition graph of (11)

$\mathrm{RR} \mathrm{n}^{\circ} 4207$ 


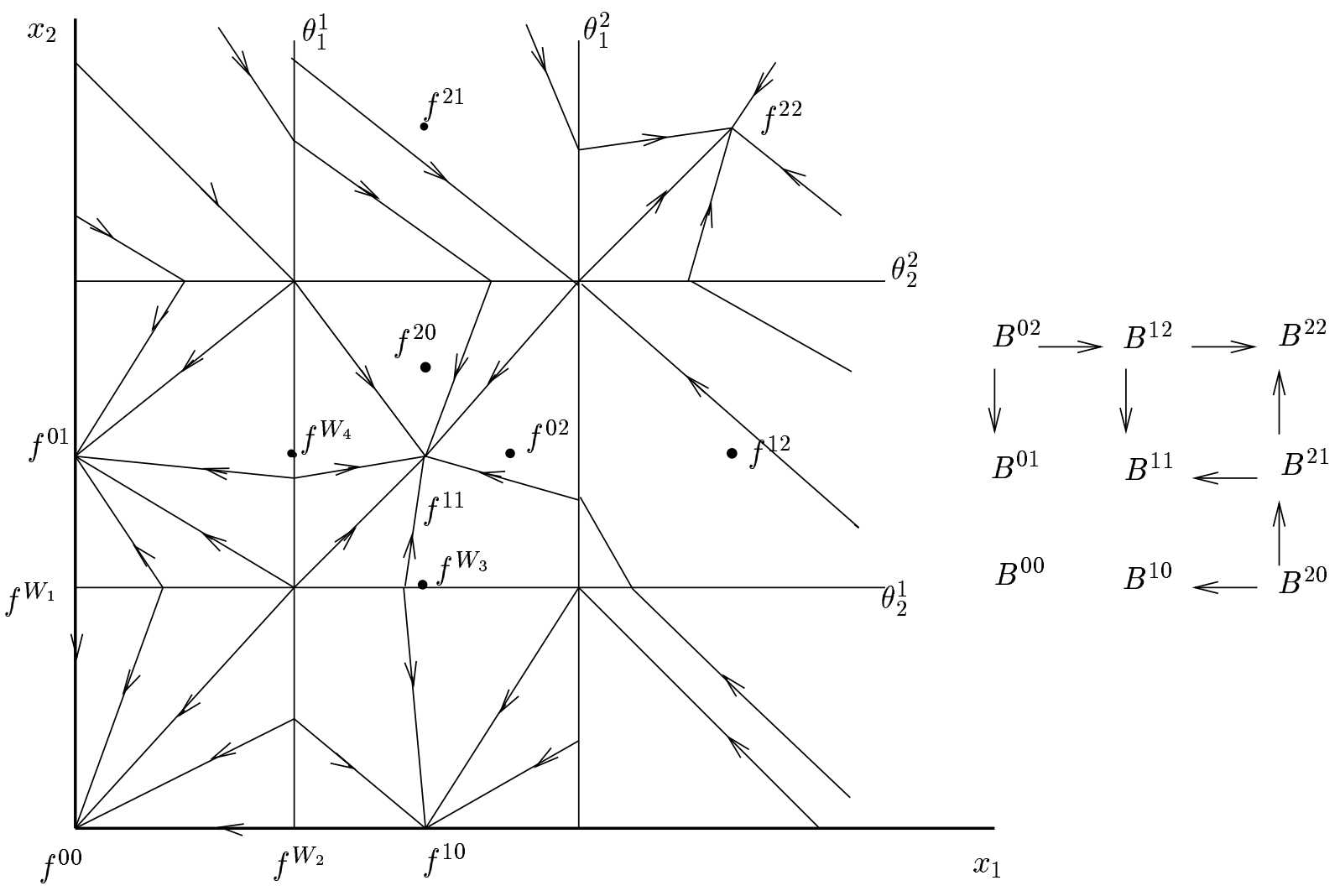

Figure 4: The flow and transition graph of (12)

INRIA 


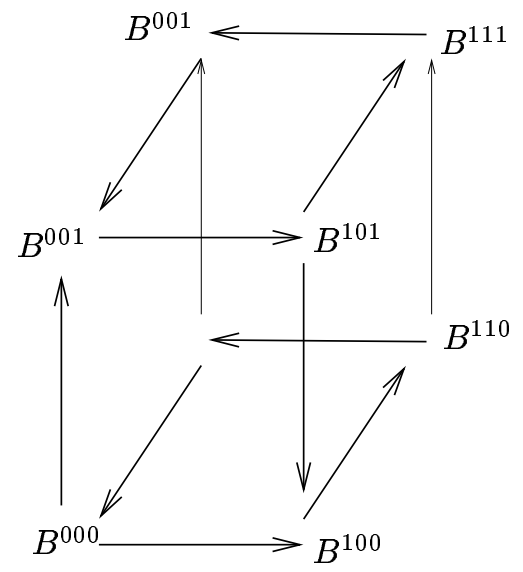

Figure 5: The transition graph of (13)
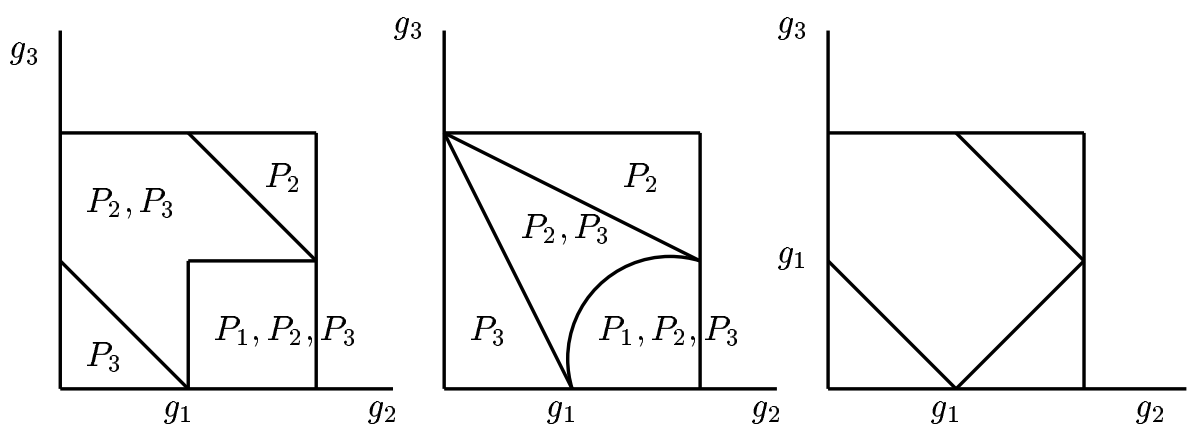

Figure 6: The domains of existence of SSP of (13 and 16), shown in the $g_{2}-g_{3}$ plane for an arbitrary value of $g_{1}$.

$\mathrm{RR} \mathrm{n}^{\circ} 4207$ 


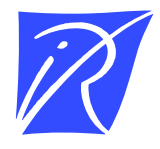

Unité de recherche INRIA Sophia Antipolis 2004, route des Lucioles - BP 93 - 06902 Sophia Antipolis Cedex (France)

Unité de recherche INRIA Lorraine : LORIA, Technopôle de Nancy-Brabois - Campus scientifique 615, rue du Jardin Botanique - BP 101 - 54602 Villers-lès-Nancy Cedex (France)

Unité de recherche INRIA Rennes : IRISA, Campus universitaire de Beaulieu - 35042 Rennes Cedex (France)

Unité de recherche INRIA Rhône-Alpes : 655, avenue de l'Europe - 38330 Montbonnot-St-Martin (France)

Unité de recherche INRIA Rocquencourt : Domaine de Voluceau - Rocquencourt - BP 105 - 78153 Le Chesnay Cedex (France)

Éditeur

INRIA - Domaine de Voluceau - Rocquencourt, BP 105 - 78153 Le Chesnay Cedex (France)

http://www.inria.fr

ISSN 0249-6399 